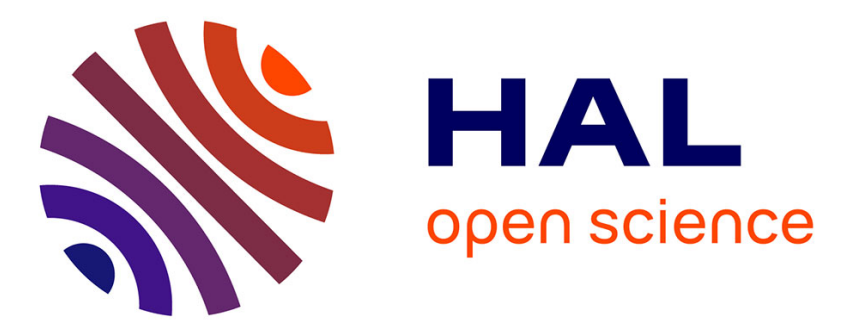

\title{
Ultrafast InGaAs photoswitch for RF signal processing
}

\author{
R. Horvath, J-F. Roux, J-L. Coutaz, Julien Poette, B. Cabon, C. Graham
}

\section{To cite this version:}

R. Horvath, J-F. Roux, J-L. Coutaz, Julien Poette, B. Cabon, et al.. Ultrafast InGaAs photoswitch for RF signal processing. IEEE International Conference on Optical Network Design and Modeling ONDM, 2017, Budapest, Hungary. hal-01958016

\section{HAL Id: hal-01958016 https://hal.science/hal-01958016}

Submitted on 12 Feb 2019

HAL is a multi-disciplinary open access archive for the deposit and dissemination of scientific research documents, whether they are published or not. The documents may come from teaching and research institutions in France or abroad, or from public or private research centers.
L'archive ouverte pluridisciplinaire HAL, est destinée au dépôt et à la diffusion de documents scientifiques de niveau recherche, publiés ou non, émanant des établissements d'enseignement et de recherche français ou étrangers, des laboratoires publics ou privés. 


\section{Ultrafast InGaAs photoswitch for RF signal processing}

\author{
R. Horvath ${ }^{1,2}$, J- F. Roux ${ }^{1 *}$, J- L. Coutaz ${ }^{1}$, \\ J. Poëtte ${ }^{2}$, B. Cabon ${ }^{2}$ \\ ${ }^{1}$ Univ. Savoie Mont-Blanc, CNRS, IMEP-LAHC, \\ Le Bourget du Lac, France \\ ${ }^{2}$ Univ. Grenoble Alpes, Grenoble-INP, CNRS, \\ IMEP-LAHC, Grenoble, France
}

\author{
C. Graham ${ }^{3}$ \\ University College of London, Department of Electronic \\ and Electrical Engineering, London, United Kingdom.
}

\begin{abstract}
Optical processing of RF signals is demonstrated in this communication using photoswitches made from Nitrogen Implanted InGaAs. The sampling device shows an ultrafast picosecond response time while activated by ultra-short optical pulses or modulated optical beam centered at the wavelength of $1.55 \mu \mathrm{m}$. The optoelectronic device is embedded in a microwave coplanar waveguide which has a high electrical bandwidth allowing to process signals in the 1-67 GHz band. We investigate the potentiality of this component to be used either in photonic assisted sampling for future Analog to Digital Converters or in photonic assisted heterodyne detection of RF modulated carriers.
\end{abstract}

Index Terms - Photoconductive sampling, Ion Implanted InGaAs, ultrafast electronics, metal-semiconductor-metal devices, Radio Over Fiber, photonic assisted data processing, heterodyne photomixer.

\section{INTRODUCTION}

$\mathrm{M}$ microwave photonics is a technology area which includes generation, processing, analog-to-digital conversion (ADC), of microwave signals using optoelectronic technological bricks such as laser, modulators, detectors and fibers. It is at the heart of future Fiber and Wireless telecommunication networks. Indeed, recent development of high performances optoelectronic components has permitted to reach the so-called $\mathrm{THz}$ communication band and wireless communications above $100 \mathrm{GHz}$ has been demonstrated, allowing higher data rate transmission [1]. In such a technological framework, the use of complex well-proven modulation schemes already used at lower frequency is challenging to reach multi-GBits/s data rate. However, such modulation requires additional signal processing at the receiver location such as heterodyne detection together with numerical data post-processing which is usually handled by specific Digital Signal Processing [2]. In order to gain performances and offer cost-effective or robust solutions it might be interesting to propose photonic assisted processing of the received RF signal modulated by the complex data stream. For instance, the use of ultralow jitter laser sources might increase the performances of the receiver by one order of magnitude as compared to full-electronics approach for ADC of $\mathrm{GHz}$ RF signals [3]. In such high bandwidth applications,

This project has received funding from the European Union's Horizon 2020 research and innovation programme under the Marie Sklodowska-Curie grant agreement No. 642355 FiWin5G. one key function is the mixing of RF and optical signals. It requires that the mixing process offers very high bandwidth together with linear responses to both the optical and electrical signals. For that it has been shown that simple MetalSemiconductor-Metal (MSM) photoswitches (PSW) made from Low-Temperature-Grown (LTG) GaAs could reach these requirements. Hence, this component has been used as ultrafast optoelectronic sampler for $\operatorname{ADC}[4,5]$ and mixer showing applications in the RF band as well as in the $\mathrm{THz}$ one $[6,7]$. Its principle rely on the increase of the electrical conductance of the semiconductor through absorption of the optical beam, making this device an optically controlled resistance. As compared to phototransistors that can also be used for such mixing, PSW do not require any DC bias to operate, and consequently may strongly reduce the DC power consumption of the mixer. Unfortunately, this kind of GaAs based component is not fully compatible with the $1.55 \mu \mathrm{m}$ telecommunication wavelength as GaAs is mostly transparent at this photon energy. In order to solve this problem, one may use InGaAs absorbing layers. Indeed up- and downconversions of an input RF signal with frequency spreading up to $20 \mathrm{GHz}$ with an optical local oscillator (LO) consisting in a modulated $1.55 \mu \mathrm{m}$ optical beam has been demonstrated [8]. Unfortunately, due to the long carrier lifetime in the InGaAs, the LO frequency was limited to $300 \mathrm{MHz}$, making this component not suitable for high data rate optoelectronic processing. Nevertheless, it is possible to strongly reduce the carrier lifetime in such semiconductor by introducing a large number of carrier trapping centers thanks to ion implantation or irradiation [9].

In this work, we investigate the use of Nitrogen Implanted InGaAs PSW that shows an ultrafast carrier response time of less than 2 ps. We demonstrate that it can be used for optoelectronic mixing and sampling of RF signal in the 1- 67 $\mathrm{GHz}$ band using lasers operating at $1.55 \mu \mathrm{m}$ : femtosecond fiber laser or self-modulating mode-locked laser diode at 60 GHz. These preliminary results open the way to more advanced studies in RF wireless communications systems in which high bit rate data streams will be sampled and/ or demodulated by optoelectronics means using such a simple, 
low-cost and robust component.

\section{DEVICE DESCRIPTION}

The sampling device has been preliminary studied in [10] where it has been shown that it possess picosecond response time and good dark resistance. In this previous work the device has been used to demonstrate simple switching experiment at frequency up to $15 \mathrm{GHz}$. The component consists in a coplanar waveguide (CPW) which central line (width $25 \mu \mathrm{m}$ ) is interrupted by a $25 \mu \mathrm{m}$ gap with interdigitated fingers as shown in the inset of Fig. 1. The fingers may be of different numbers (3 to 5) and their separation is consequently varying from 3 to $5 \mu \mathrm{m}$. The CPW is about $750 \mu \mathrm{m}$ long and is made from gold electrodes deposited on a Fe:InP substrate. At the location of the $25 \times 25$ $\mu \mathrm{m}^{2}$ gap, a $300 \mathrm{~nm}$ thick mesa of InGaAs was deposited onto the Fe:InP substrate. The device is then irradiated with Nitrogen atoms that create a large number of defects in the InGaAs layer and then penetrate deeply in the Fe:InP substrate. Considering the implantation parameters the concentration of EL2 type defects is estimated to be around 5 $10^{17} \mathrm{~cm}^{-3}$ ensuring a picosecond response time. In the following sections all the measurements were done "on wafer" using RF electrical coplanar probes.

\section{DEVICE CHARACTERIZATION}

As mentioned above, the electrical characteristics of the devices (I-V curve and dark resistance) have already been measured in [10] and we have checked that most of the samples have kept similar characteristics. We then investigate in more details the high bandwidth properties of the PSW in terms of electrical and optoelectronical bandwidth. Before that we want to remind the basic operation of such an ultrafast PSW when illuminated by an optical beam. In the dark state, the lumped electrical equivalent model of the PSW is a capacitance $\mathrm{C}$ in parallel with a dark resistance Rdark (see the inset of Fig. 2). When illuminated by an optical beam with photon energy above the bandgap of the InGaAs layer, the conductance increase due to the photo-generation of free carriers in the absorbing layer. In a first approximation, the conductance varies linearly with the absorbed optical power. Its value depends on the geometry of the switch and also on the dynamics of the photogenerated carriers and their electrical properties such as mobility. In [5 and 6] we have studied the behavior of an ultrafast photoswitch illuminated either by femtosecond optical pulses or by amplitude modulated optical beams. In both cases, we have demonstrated that the temporal behavior of the device is mainly governs by the carrier lifetime of the electrons that can be roughly approximate by an exponential law $\mathrm{e}^{-\mathrm{t} / \mathrm{\tau e}}$ where $\tau_{\mathrm{e}}$ is the carrier lifetime of the photogenerated electrons.

In order to investigate the dynamics of the optoelectronic response of the samples, we have performed optoelectronic autocorrelation [11]. This experiment consists in illuminating the photoswitch with two femtosecond pulses that are delayed by $\Delta t$ one from each other thanks to an optical delay line while the PSW is DC biased and loaded by a $50 \Omega$ resistor. The delay, $\Delta \mathrm{t}$, is continuously varied and we record the average voltage observed at the load resistor. Because the PSW acts as a voltage divider with the load resistor the overall circuit shows a non-linear response to the optical pump excitation. Consequently, as compared to the case where the two exciting pulses are separated by a time much larger than the PSW response time $\left(\Delta \mathrm{t}>\tau_{\mathrm{e}}\right)$, the autocorrelation signal is decreasing as long as the time delay in between the two pulses is reduced: the autocorrelation signal is minimal at $\Delta \mathrm{t}=0$. Moreover, the temporal behavior of the optoelectronic autocorrelation trace gives a direct insight about the PSW temporal dynamics if the duration of the exciting optical pulses is much shorter than $\tau_{\mathrm{e}}$.

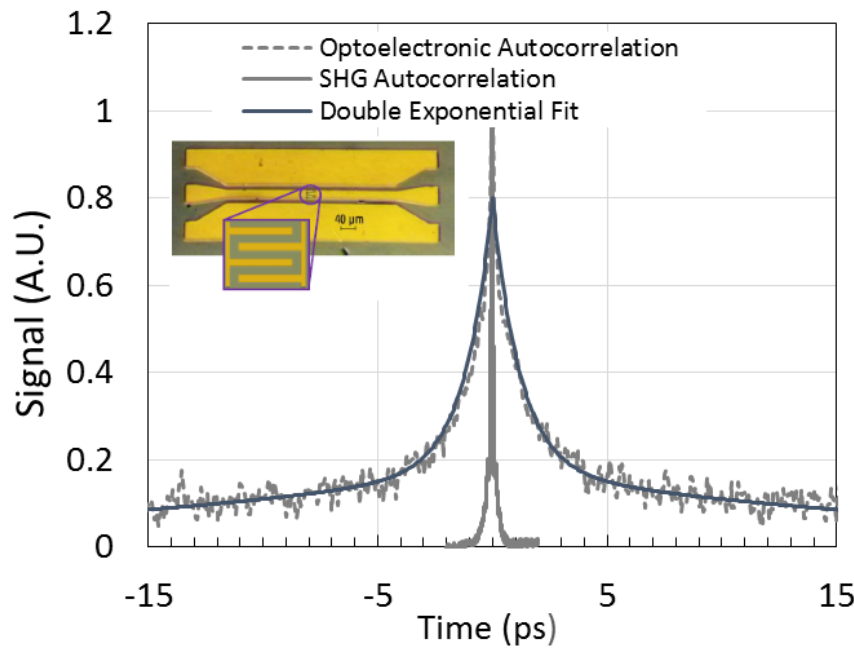

Fig. 1. Optoelectronic (dashed grey line) and optical (grey line) autocorrelation traces of a Nitrogen implanted InGaAs PSW (inset). A double exponential fitting curve with time constants of .2 and 20 ps is also shown (black line).

Here we perform the experiment with a fiber femtosecond laser operating at $1.55 \mu \mathrm{m}$ (MENLO C-Fiber) with a repetition rate of $100 \mathrm{MHz}$. The two delayed optical pulse trains are propagating toward the switch through 3 meter of SMF 28 fiber which linear group velocity dispersion is compensated by a section of DCF 38 fiber (THORLABS DCF 38). We checked that the duration of the optical pulses at the output of the fibers is limited to less than 100 fs by measuring the optical autocorrelation trace thanks to Second Harmonic Generation in a BBO non-linear crystal. This optical autocorrelation trace is plotted in Fig. 1 (continuous grey line) it gives also the zero delay $(\Delta \mathrm{t}=0)$ time of the experimental set-up. Finally we record the optoelectronic autocorrelation signal for average optical power of 1.5 and $2.5 \mathrm{~mW}$ for each of the two delayed beams. A typical result is plotted in Fig. 1 (dashed grey line) it consists in a double side exponential function and shows a very sharp peak around zero delay which is a signature of some optical interferences between the two optical pulses. Ignoring this coherence peak, the optoelectronical autocorrelation trace is fitted with the formula: $A_{1} \exp \left(-t / \tau_{1}\right)+A_{2} \exp \left(-t / \tau_{2}\right)$. We found the best fit for $\mathrm{A} 1=0.6, \mathrm{~A} 2=0.2$ and $\tau_{1}=1.2 \mathrm{ps}$ while $\tau_{2}=20$ ps (black line). These time constants confirm the picosecond response time of these nitrogen implanted InGaAs PSW [10]. Here we show 
that the 4.4 ps response time that was observed in [10] with an ultrafast oscilloscope was certainly limited by the bandwidth of the electronic sampling heads. From the 1.2 ps response time, we expect a cutoff frequency of the device larger than $100 \mathrm{GHz}$. The longer $20 \mathrm{ps}$ time constant that we observe may either be attributed to a contribution of holes or to an electron de-trapping time; its lower amplitude reduces its impact on the overall optoelectronic bandwidth of the PSW.

In a second experiment, we have checked the electrical bandwidth of the device by measuring its $\mathrm{S}$ parameters in the dark state using a $0.04-65 \mathrm{GHz}$ Vector Network Analyzer (VNA). The experimental frequency evolution of the $\mathrm{S}_{12}$ parameter is plotted in Fig. 2 together with the theoretical curve obtained considering the basic lumped element equivalent model of the gap. We found an equivalent capacitance of $5.7 \mathrm{fF}$ and a dark resistance of $5 \mathrm{k} \Omega$. This low capacitance, also insure an ultrafast $\mathrm{RC}$ response time shorter than 300 fs for a $50 \Omega$ load impedance.

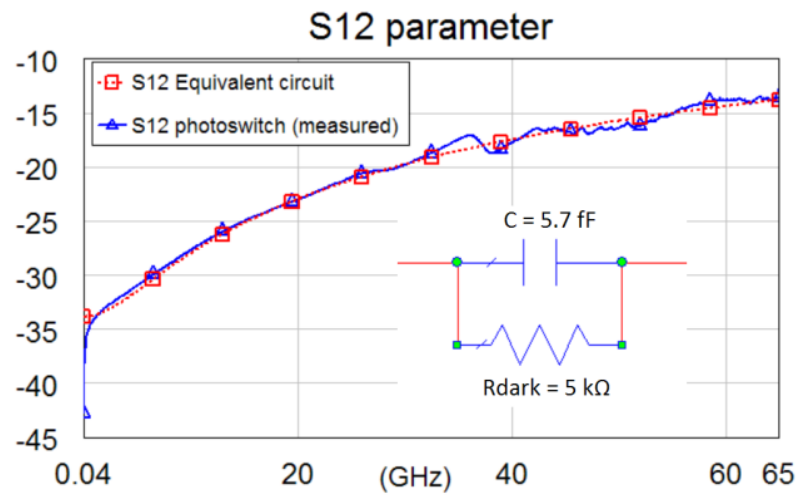

Fig. 2. S21 parameter of the PSW in the dark state. The inset shows the lumped elements equivalent circuit of the device.

\section{PHOTOCONDUCTIVE SAMPLING OF RF SIGNALS}

After characterization of the fabricated structures we have used them to demonstrate their ability of sampling RF signals. Here we have chosen an asynchronous sampling experiment in which the RF signal is free running while the sampling frequency is fixed by the repetition rate of the femtosecond laser $\left(F_{\text {laser }}=100 \mathrm{MHz}\right)$. The electrical input of the PSW is directly excited by the AC output of an Agilent RF Synthesizer E8257 D working in the $250 \mathrm{kHz}-67 \mathrm{GHz}$ band with enhanced power mode (up to $+15 \mathrm{dBm}$ ). In this experiment, the PSW is not DC biased but it is optically activated by the train of fs pulses delivered by the femtosecond laser already described above. The RF output signal of the PSW is characterized with a spectrum analyzer (Anritsu MS2667C of $30 \mathrm{GHz}$ bandwidth). As shown in [5] when excited by an RF signal of frequency $F_{R F}$, the illuminated PSW delivers a train of electrical pulses at the repetition rate $F_{\text {laser }}$, which amplitude depends on the amplitude of the RF signal, the optical power and the conversion efficiency of the switch. In the frequency domain, the electrical output consists in a Dirac-comb centred on the RF angular frequency $F_{R F}$ with peaks separated by the frequency $F_{\text {laser }}$ of the laser pulses train:

$$
S(f)=\sum_{n=-\infty}^{n=+\infty} S_{n}(f) \times \delta\left(f-f_{R F}-n f_{\text {laser }}\right)
$$

The factor $S_{n}(f)$ is the amplitude of the $\mathrm{n}^{\text {th }}$ harmonics of the frequency comb. It depends on the conversion efficiency of the switch which is decreasing when the RF frequency is above the cutoff frequency of the device. For RF input signals in the range 1 to $67 \mathrm{GHz}$, we measured the power of the $n^{\text {th }}$ harmonic using a $80 \mathrm{~dB}$ amplification stage with a $1 \mathrm{GHz}$ bandwidth. So, we choose the $\mathrm{n}^{\text {th }}$ harmonic which falls in the vicinity of $15 \mathrm{MHz}$. For example, if the input RF signal frequency is $30.015 \mathrm{GHz}$, the output of the PSW consists in a Dirac Comb centered around $30.015 \mathrm{GHz}$ with harmonics every $100 \mathrm{MHz}$. The $300^{\text {th }}$ harmonic of the sampled signal is positioned at $15 \mathrm{MHz}$ and is thus amplified by $40 \mathrm{~dB}$.

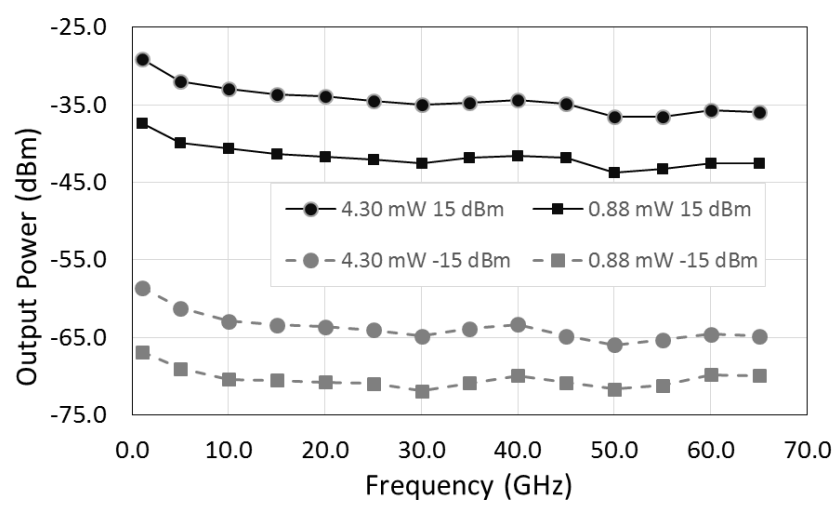

Fig. 3. Output power of the sampled signal for different experimental conditions: average optical power of 4.3 or $0.88 \mathrm{~mW}$ and RF power of - 15 $\mathrm{dBm}$ or $+15 \mathrm{dBm}$. The $\mathrm{x}$ axis represents the frequency of the RF input signal.

The results, compensated with the losses of the RF cables are plotted in Fig.3. We observe that the amplitude of the sampled signal shows a small frequency roll-off. Actually, depending on the experimental conditions we observe only 4 to $6 \mathrm{~dB}$ of decrease in between the amplitude measured at 1 $\mathrm{GHz}$ and that measured at $67 \mathrm{GHz}$. This confirm the large bandwidth of the device. Moreover, by varying the input RF power from - $15 \mathrm{dBm}$ to $15 \mathrm{dBm}$ we checked that the sampler output amplitude depends linearly on the input power. On the other hand we observe that the amplitude of the RF output increase by approximately $10 \mathrm{~dB}$ when the optical power is increased by $7 \mathrm{~dB}$ (from 0.88 to $4.3 \mathrm{~mW}$ ). This means that the RF output power varies approximately with the 1.5 power of the optical power, although this dependence should be, in principle, quadratic for low illuminating power.

\section{HETERODYNE DEMODULATION OF AMPLITUDE MODULATED RF SIGNALS}

Another potential application of such ultrafast PSW is modulation or demodulation of RF signals with optical signals using the PSW as a hybrid (optical / electronic) mixer [6, 7]. Here we have investigated the potentiality of the InGaAs PSW to perform this operation in an experiment which is very similar to the previous one. Here, the optical signal consists in a $1.55 \mu \mathrm{m} \mathrm{CW}$ beam modulated at a frequency $F_{L O}$ and the RF input signal is at a frequency $F_{R F}$. These signals are mixed 
within the PSW, the flow of photocarriers that are generated by the absorption of the $1.55 \mu \mathrm{m}$ optical beam being accelerated by the RF electrical field applied to the PSW. This mixing results in an RF output signal at the difference frequency $F_{I F}=F_{R F}-F_{L O}$. Here again the RF frequency varies from 1 to $65 \mathrm{GHz}$ while the RF power varies from -15 to +15 $\mathrm{dBm}$. As a modulated laser beam, we used an InAs/InP-based quantum dash mode locked laser diode self-oscillating at $58.625 \mathrm{GHz}$ [12]. In order to compensate for the laser dispersion we use $70 \mathrm{~m}$ of SMF28 monomode fiber and a variable optical attenuator allows to vary the average optical power used to excite the PSW. The RF output of the PSW is detected thanks to a RF Spectrum Analyzer (RFSA) (Anritsu MS2668C $9 \mathrm{kHz}-40 \mathrm{GHz}$ ). For RF input frequency in the range $19-67 \mathrm{GHz}$, the IF frequency falls within the allowed detection band. In Fig. 4 we have plotted the amplitude of this IF signal for an input RF power of $15 \mathrm{dBm}$. For an optical power of $5 \mathrm{~mW}$, the IF RF power is about $-65 \mathrm{dBm}$ leading to an estimated losses $\mathrm{P}_{\mathrm{IF}} / \mathrm{P}_{\mathrm{RF}}$ of $80 \mathrm{~dB}$. We note that these large losses are within the same order of magnitude than the ones presented by Unbiased Ultrafast Photodiodes used as Optoelectronic mixer [13] and might been strongly reduce by smart engineering of the photoconductive device [7]. Similarly to the ultrafast photodiodes from [13], our PSW presents an ultra large bandwidth of more than $65 \mathrm{GHz}$ as confirm by its constant response over the whole frequency range except in the vicinity of $58 \mathrm{GHz}$ where a systematic experimental decrease, which is under investigation, is observed. Finally, we have experimentally confirm the linear response of the photomixer versus the RF input power and we have demonstrated that the losses of the mixer might be reduced by increasing the impinging optical power (inset of Fig. 4).

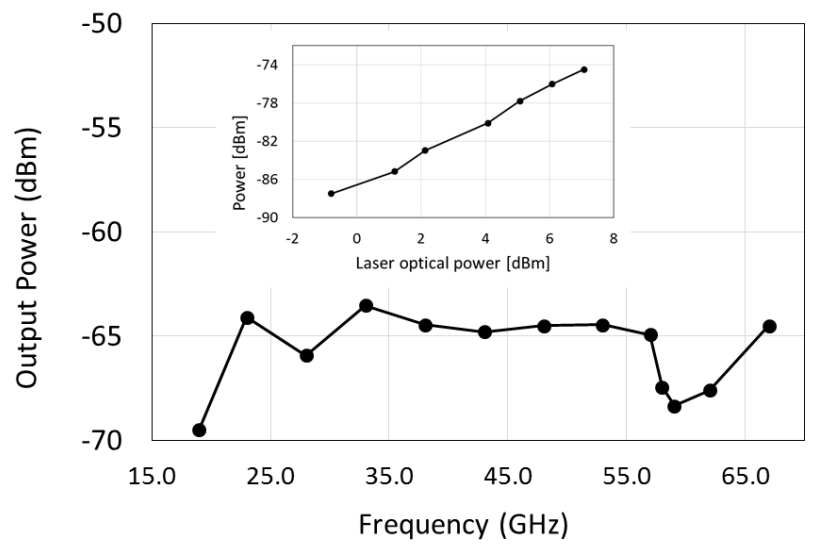

Fig. 4. Power of the IF signal for different input RF frequencies. The PSW is used as a heterodyne mixer in between a $14 \mathrm{dBm} R \mathrm{RF}$ input signal and a 5 $\mathrm{mW}$ optical beam self-oscillating at $58.625 \mathrm{GHz}$. The inset shows the IF power dependence onto the optical power.

\section{CONCLUSION}

In this work we have presented preliminary results of photoconductive sampling and mixing of RF signals using Nitrogen implanted InGaAs based CPW structures. The device is characterized by $5 \mathrm{kOhms}$ dark resistance and low equivalent capacitance. Its optoelectronic response shows a very fast recovery time of 1.2 ps that allows us to demonstrate sampling of $67 \mathrm{GHz}$ signals. Moreover we have performed heterodyne mixing in between an RF signal of frequency up to $67 \mathrm{GHz}$ and an optical beam self-modulated at $58.625 \mathrm{GHz}$. These first demonstrations of principle are opening the way to demodulation and photonic assisted processing of high bit rate data streams carried either by the RF or by the optical signal.

\section{ACKNOWLEDGMENT}

We would like to thank Mr S. Formont from THALES TSA for providing us RF amplifiers and filters and $\mathrm{Mr} \mathrm{N}$. Corrao, from IMEP-LAHC for his valuable help in preparing the RF experiments.

\section{REFERENCES}

[1] A. Seeds, H. Shams, M. Fice and C. Renaud, "TeraHertz Photonics for Wireless Communications," IEEE J. Lightwave Technol., vol. 33, pp. 579-587, 2015.

[2] F. Lecoche, E. Tanguy, B. Charbonnier, Hongwu Li, F. van Dijk, A. Enard, F. Blache, M. Goix, and F. Mallécot, "Transmission Quality Measurement of Two Types of $60 \mathrm{GHz}$ Millimeter-Wave Generation and Distribution Systems," IEEE J. Lightwave Technol., vol. 27, pp. 5469-5474, Dec. 2009.

[3] A. Khilo, S. J. Spector, M. E. Grein, A. H. Nejadmalayeri, C. W. Holzwarth, M. Y. Sander, M. S. Dahlem, M. Y. Peng, M. W. Geis, N. A. DiLello, J. U. Yoon, A. Motamedi, J. S. Orcutt, J. P. Wang, C. M. Sorace-Agaskar, M. A. Popović, J. Sun, G.-R. Zhou, H. Byun, J. Chen, J. L. Hoyt, H. I. Smith, R. J. Ram, M. Perrott, T. M. Lyszczarz, E. P. Ippen and F. X. Kärtner, "Photonic ADC: overcoming the bottleneck of electronic jitter,” Opt. Exp., vol. 20, pp. 4454-4469, 2012.

[4] L. Y. Nathawad, R. Urata, B. Wooley and D. A. B. Miller, "A 40-GHzbandwidth, 4 bit, time-interleaved A/D converter using photoconductive sampling," IEEE J. Solid-State Circuits, vol. 38, pp. 2021-2030, Dec. 2003.

[5] J.-F. Roux, J.-M. Delord, and J.-L. Coutaz, "RF Frequency Response of Photoconductive Samplers," IEEE J. Quantum Electron., vol. 47, pp. 223-229, Feb. 2011

[6] M. B. Kuppam, J.-F. Lampin, E. Peytavit, J. -F. Roux, and J. -L. Coutaz, "Study of Ultrafast Semiconductor Photoswitches for CW RF Signal Sampling and Modulation », IEEE J. Lightwave Technology, vol. 32, Issue 20, pp. 3839-3845, 2014.

[7] E. Peytavit, F. Pavanello, G. Ducournau and J.-F. Lampin, "Highly efficient terahertz detection by optical mixing in a GaAs photoconductor,” Appl. Phys. Lett., vol. 103, 201107, 2013.

[8] H.-J. Song, T.-W. Kim, S. J. Jo, C.-H. Lim, K.-H. Oh, S.-G. Ihn, and J.I. Song, "Microwave Photonic Mixer Utilizing an InGaAs Photoconductor for Radio over Fiber Applications," IEICE Trans. Electron., vol. E90, p. 457, 2007.

[9] C. Carmody, H. H. Tan, C. Jagadish, A. Gaarder and S. Marcinkevicius, "Ion-implanted In0.53Ga0.47As for ultrafast optoelectronic applications", Appl. Phys. Lett., vol. 82, pp. 3913- 3915, June 2003.

[10] C. Graham, R. Gwilliam, and A. Seeds, "Nitrogen ion implanted InP based photo-switch," Opt. Express, vol. 20, 26696-26703, 2012.

[11] J.-M. Delord, J.-F. Roux, J.-L. Coutaz, and N. Breuil, "Study of optoelectronic sampler linearity for analog-to-digital conversion of RF signals," IEEE Photon. Technol. Lett., vol. 21, pp. 1369-1371, Oct. 2009.

[12] F. Brendel, J. Poëtte, B. Cabon, T. Zwick, F. van Dijk, F. Lelarge, and A. Accard, "Chromatic Dispersion in $60 \mathrm{GHz}$ Radio-Over-Fiber Networks Based on Mode-Locked Lasers," J. Lightwave Technol. vol. 29, pp. 3810-3816, 2011.

[13] E. Rouvalis, M. Fice, C. Renaud, and A. Seeds, "Optoelectronic detection of millimetre-wave signals with travelling-wave uni-travelling carrier photodiodes," Opt. Express vol. 19, 2079-2084, 2011. 